\title{
Analysis by x-ray microtomography of a granular packing undergoing compaction
}

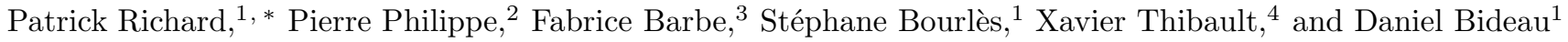 \\ ${ }^{1}$ G.M.C.M., UMR CNRS 6626, Université de Rennes I, F-35042 Rennes Cedex, France \\ ${ }^{2}$ P.M.M.H., UMR CNRS 7636, ESPCI, 10 rue Vaucquelin, F-75231 Paris cedex 05, France \\ ${ }^{3}$ L.M.R., UMR 6138, INSA Rouen, Campus du Madrillet, \\ F-76801 Saint-Etienne-du-Rouvray Cedex, France \\ ${ }^{4}$ ESRF, BP 220, F-38043 Grenoble Cedex, France
}

(Dated: today)

\begin{abstract}
Several acquisitions of X-ray microtomography have been performed on a beads packing while it compacts under vertical vibrations. An image analysis allows to study the evolution of the packing structure during its progressive densification. In particular, the volume distribution of the pores reveals a large tail, compatible to an exponential law, which slowly reduces as the system gets more compact. This is quite consistent, for large pores, with the free volume theory. These results are also in very good agreement with those obtained by a previous numerical model of granular compaction.

PACS numbers: 45.70.Cc, 41.60.Ap
\end{abstract}

Understanding the slow dynamics of out-of-equilibrium systems is still an open and debated issue. Even though granular media are not thermal systems, due to the insignificance of the thermal energy $k_{B} T$ in comparison to the energy needed to move a macroscopic grain, they are of great interest in this context. Indeed, it has been suggested that the relaxation of a granular medium under weak mechanical perturbations, such as shaking or shearing, has a formal analogy with the slow dynamics of out-of-equilibrium thermal systems [1, 22. This analogy is based on the idea that the geometry of the system is more important than any other parameter as the driving energy or the interaction between particles. Consequently, granular media are often presented as ideal systems for studying the relaxation towards equilibrium [3]. Nevertheless, one may keep in mind that, unlike the thermal energy, the mechanical agitation of the granular material is neither stochastic non generally isotropic and thus leads to different spatial scales of the energies [4].

One of the most common behavior of a shaken granular packing is its slow compaction. This compaction is easily observed through the progressive increase of the volume fraction and appears to be a simple way to investigate the above analogy. The first experiments on compaction have been realized in a thin tube giving rise to a high lateral steric constraint in the packing $[5]$. The evolution of the volume fraction reveals a very slow dynamics and can be reasonably well fit by an empirical law in the inverse of the logarithm of the number of shakes. More recently, Philippe and Bideau carried out new experiments with a reduced lateral confinement [6]. They showed that the resulting compaction dynamics is consistent with a stretched exponential, also called Kholrausch-WilliamsWatts function, which is the most usual description of the relaxation in a out-of-equilibrium system such as a glass (see for instance [7] and references therein).

*Electronic address: Patrick.Richard@univ-rennes1.fr
Contrary to most previous studies on granular compaction in which the quantities measured deal with the properties of, at least, a part of the packing (as the mean packing fraction $\Phi[\underline{5},[\underline{6}$ or the vertical profile $[\underline{6}]$ ), the study discussed here benefits from x-ray microtomography to characterize the microstructure of a packing undergoing compaction. A previous work on x-ray microtomography applied to granular materials has been carried out recently that involves a structural study of a unique packing of about 2000 nearly monosized beads [8]. In this paper we focus on the microstructural evolution of a compacting granular bed by means of an original statistical analysis and we relate this to the free volume theory.

We use an experimental setup close to previous ones [5, 6] : $200-400 \mu \mathrm{m}$ diameter glass beads are poured to about $80 \mathrm{~mm}$ height in a $8 \mathrm{~mm}$ inner-diameter glass cylinder. The whole is vertically shaken by sinusoidal excitation at a frequency of $70 \mathrm{~Hz}$. The applied acceleration is measured by an accelerometer and the intensity of the vibration is characterized by $\Gamma$, the maximal applied acceleration normalized by gravity $\left(g=9.81 \mathrm{~ms}^{-2}\right)$. The experiments are performed as follows: starting from an initial loose reproducible configuration $(\Phi \approx 0.57)$, a packing is vibrated for a given number $N$ of oscillations with a fixed acceleration $\Gamma$ and then analyzed by x-ray microtomography. Experimental data acquisitions have been recorded at the ID19 beam line of the ESRF (European Synchrotron Radiation Facilities). Synchrotron radiation improves upon traditional $\mathrm{x}$-ray techniques and offers alternative methods of imaging. State-of-the-art microtomography provides the three-dimensional mapping of the linear absorption coefficient in the bulk of millimeter-sized samples with a spatial resolution of the order of $1 \mu \mathrm{m}$. A description of this experimental method may be found in [9]. A monochromatic coherent beam is used to get sample radiography for 1200 angular sample positions ranging from $0^{\circ}$ to $180^{\circ}$. An x-ray energy of $51 \mathrm{keV}$ was selected to ensure a high enough signal to noise ratio. The exposure time is $1 \mathrm{~s}$ by projection. The energy is selected using a classical double monocrystal 


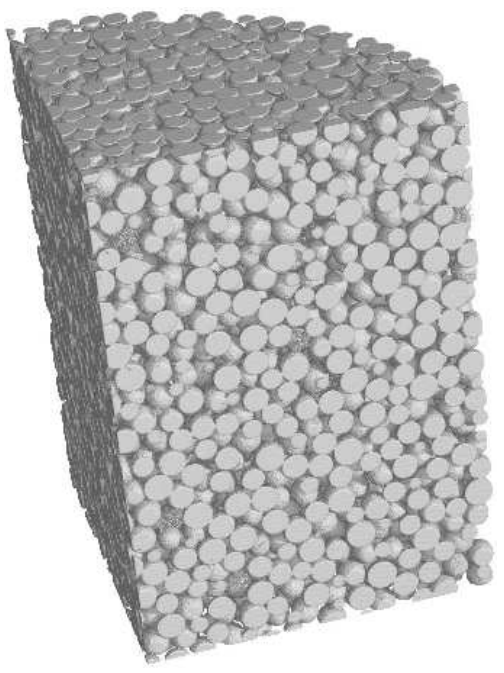

FIG. 1: Part of the 3D reconstruction of the initial packing $(\Phi \approx 0.57)$.

device. A filtered backprojection algorithm 10, 11] is used to compute the three-dimensional mapping of the linear absorption coefficient in the sample. The light detector used is based on the the fast read out low noise charge couple device (FRELON CCD) camera developed by the ESRF detector group $(1024 \times 1024$ elements, 14 bits dynamic). A thin scintillation layer deposited on glass converts x-rays to visible light. Light optics magnify the image of the scintillator and project it onto the CCD. With such a setup, the resolution is $9.81 \mu \mathrm{m}$ by pixel. Each reconstructed packing contains around 15000 grains. An example is reported in Fig 1 .

Using an image processing software, the size and the location of each particle are recovered. Then a complete set of information is available to study the geometry of the packing. In the following we note $\langle R\rangle$ and $\langle w\rangle$ the mean radius and mean volume of a sphere, respectively.

The first characteristics reported are the classical pair correlation function $g(r)$. Figure2(a) represents the variation of the pair correlation function before and after more than $10^{4}$ excitations of intensity $\Gamma=3.0$. Note that the initial packing fraction, measured with the image processing software, is equal to 0.57 , whereas the steadystate packing fraction, obtained after a large number of excitations, is approximately 0.62 . It appears that the different peaks of $g(r)$ slowly move with the number of taps. In particular the second peak slightly progresses from $r=4\langle R\rangle$ to $r=2 \sqrt{3}\langle R\rangle$, which is characteristic of developing structure. Besides, one can also remark that unlike Seidler et al. 8], we do not observe a double peak around $r=4\langle R\rangle$. This is due to large size distribution of the grains in our experimental work (in [8] it is significantly weaker.) Nevertheless the evolutions observed for $g(r)$ are not significant. It is even more striking if one compares $g(r)$ for steady-states corresponding to different intensities of excitation as reported in Fig 2(b). Here the steady-state pair correlation functions obtained for
$\Gamma=0.95,1.6$ and 3.0 have been plotted; their packing fractions are, respectively, $0.617,0.639$, and 0.624 . These correlation functions do not differ significantly. It means that the pair correlation function is not a suitable tool to study structural changes during granular compaction. Compaction is then a process more subtle than a simple decrease of the mean distance between grains. Since this function is averaged over all the angles of orientation, angular reorganizations cannot be seen. In contrast to the numerical work of Rosato and Yacoub [12], we do not observe the appearance of secondary sharp peaks in the pair correlation function. This may be due to the difference in terms of vibration intensity $\Gamma$ (up to 3 in this work, 6.4 in [12]) and to the small size of the vessel they used, which can induce strong wall effects as crystallization. Note that by using a local bond order parameter [13] we do not observe crystallization due to the walls. This is not surprising since the size distribution of our grains is rather large.

Further evidence of a transformation in the packing microstructure is provided by the study of the size of the interstitial voids. This can also be an experimental test for the free volume theory [4, 14] that postulates an exponential decay for the distribution of these voids $\rho(v) \propto \exp \left(-v / v_{0}\right)$. Following previous works 15, 16] we define a pore size via the Voronoi tessellation. A Voronoï polyhedron around a sphere is the region of space in which all points are closer to this sphere than to any other sphere in the packing. The Voronoï network that is the whole collection of the vertices and of the edges of the polyhedra maps the pore space. Each vertex of this tessellation is equidistant to four neighboring spheres and therefore defines the center of a pore. The volume of a pore is then the size of a virtual sphere centered on the vertex and in contact with the four neighboring spheres. The volume of this void-sphere partially reflects the volume of the whole void situated inside the tetrahedron formed by the centers of four neighboring spheres. Since in our experiments the spheres are not perfectly monosized, we have adapted this method to a polydisperse packing replacing the Voronoï tessellation by the so-called navigation map [17]. It is then possible to compute the size distribution of the pores for each packing. Following Philippe and Bideau [16] the volume of the pores $v$ is normalized by the mean volume of a grain $\langle w\rangle$. Figure [3 presents the evolution of the volume distribution of the pores for $\Gamma=3.0$ at different stages of the compaction. First of all, we observe that unlike the pair correlation function, $\rho(v /\langle w\rangle)$ drastically changes with the number of excitations. Indeed an exponential decay law is found for the distribution of the voids, at least for the pores larger than octahedral pores $\left(v>(\sqrt{2}-1)^{3} \approx 0.0711\right)$. The exponential shape persists during the compaction of the packing yet with a reduction of the tail, i.e. with a decrease of the characteristic volume $v_{0}$. A typical evolution of $v_{0}$ is shown in the inset of Fig 3(a). As reported in Fig 3(b), the final exponential decays of 

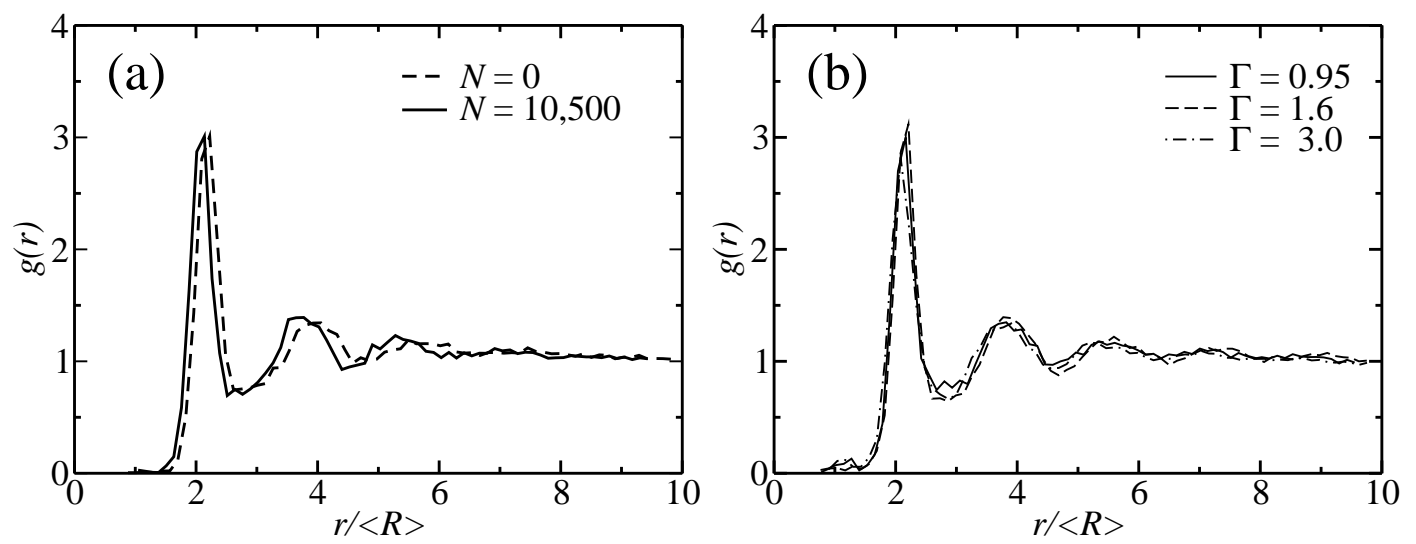

FIG. 2: (a) Evolution of the pair correlation function for $\Gamma=3.0$. (b) Steady-state pair correlation functions obtained for $\Gamma=0.95, \Gamma=1.6$ and $\Gamma=3.0$.
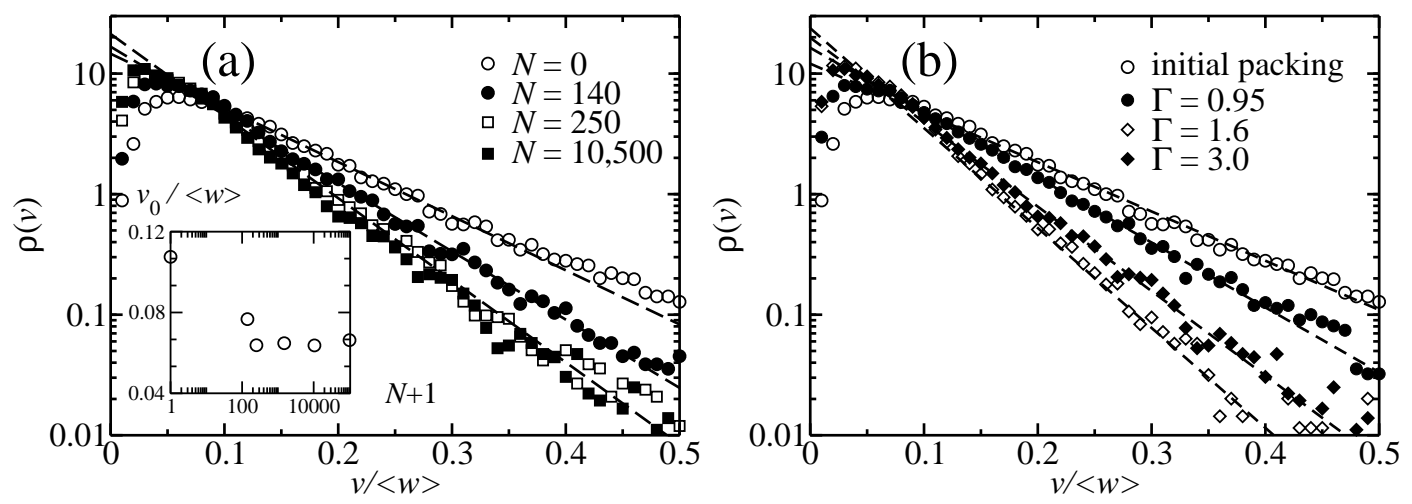

FIG. 3: (a) Evolution of the volume distribution of the pores during compaction with $\Gamma=3.0$. Inset of (a): decrease of $v_{0} /\langle w\rangle$ with the number of oscillations $N$ and for $\Gamma=3.0$. (b) Volume distributions of the pores for the initial packing and for three different steady-state packings obtained for $\Gamma=0.95,1.6$ and 3.0 (b).

the steady-state distribution, obtained after a sufficient number of oscillations, are strongly dependent on the intensity $\Gamma$ of the vibrations. By contrast, the part of the distribution corresponding to the smallest pores is less $\Gamma$ dependent. The remarkable point is that these results are very close to those obtained by numerical simulation by Philippe and Bideau [16]. Indeed, as is illustrated in Fig. [4 they also found an exponential decay for $\rho(v /\langle w\rangle)$ which slightly reduces as the system compacts. Their simulation is solely based on the geometric constraints in a three-dimensional packing of identical hard spheres. Each tap is decomposed into a vertical dilation of intensity $\epsilon(z \rightarrow z[1+\epsilon])$ followed by a nonsequential deposition. This last stage is ruled by gravity (the individual motions of the particles are preferentially oriented along the vertical) and by the condition of non interpenetration between particles. When the gravitational energy of the whole packing gets nearly stabilized, the deposition is stopped and another tap is implemented. More details can be found in [16]. The agreement between this simulation and our experiments emphasizes the fundamental importance of the geometry in granular compaction. The steric constraint between the grains seems to rule the local rearrangements of grains allowed by the shaking energy. One can observe that the small pore distributions for the numerical simulations and the experiments are quite different. We explain this point by the fact that contrary to our experiments the grains used for the numerical simulations are perfectly monosized and moreover they are never in real contact (see [16].) Indeed the grain size distribution decreases the mean volume of the pores and therefore the number of small pores is larger in the experiments. We can also observe that the existence of an exponential tail does not seem to be sensitive to the polydispersity. However, we should point out that contrary to our experiments and to previous work [ 6 , significant packing fraction gradients are present in the simulations. They are probably consequences of the $z$-dependent dilation used in the model. In order to quantify the importance of the tail in the pore size distribution we also have plotted on the inset of Fig. 目 $v_{0} /\langle w\rangle$ as a function of $1 / \Phi$ for experiments and numerical simulations, for any available number of taps and any shaking acceleration. We observe a linear correlation between the two quantities which is 


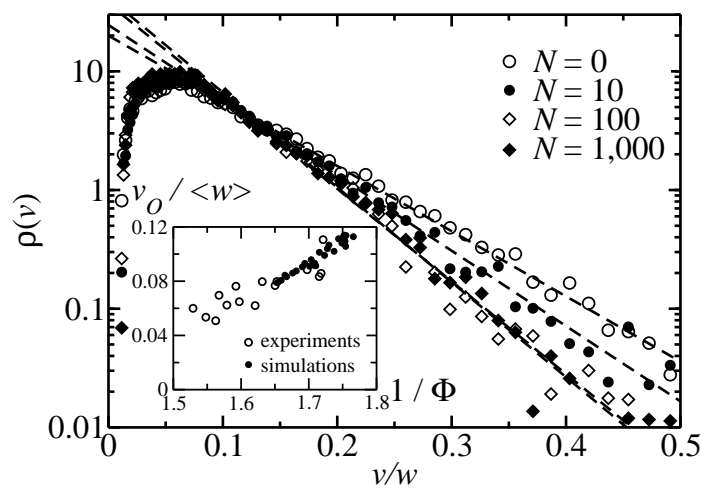

FIG. 4: Volume distributions of the pores obtained with the numerical model of [16] at different stages of a packing undergoing compaction with the dilation parameter $\epsilon=0.1$. Here $w$ is the volume of the particles. Inset : plot of $v_{0} /\langle w\rangle$ as a function of $1 / \Phi$ for all experiments (o) and a fully representative set of simulations $(\bullet)$.

in agreement with the free volume theory [4, 14]. This curve also shows that the packing fraction $\Phi$ and the characteristic volume $v_{0}$ vary in the same way during the compaction.

As demonstrated here, the use of X-ray microtomography to study granular compaction provides much information on the microstructure of a granular packing undergoing compaction. This further opens several perspectives to this work. First of all, our analysis gives an experimental measure of the free volume and can be used as an experimental test of the free volume theory for granular compaction $([4,14])$. Then, a quantitative comparison between these microtomography experiments and the numerical simulations described above can be done. In particular, we plan to correlate the experimental excitation parameter $\Gamma$ with $\epsilon$, the numerical one. For that purpose we will use the measure of the characteristic volume of a pore $v_{0}$ and assume this quantity provides the link between $\Gamma$ and $\epsilon$. We also plan to study the hysteresis on the steady-states and the microstructure of a granular material during the compaction along the well-known reversible and irreversible branches [18].

In this article, we have reported experimental results on the development of the microstructure in a granular packing submitted to sinusoidal vibrations. It has been shown that, even at rather high acceleration $(\Gamma=3)$, no significant changes are observed in the pair correlation function. The structural signature of compaction is then more subtle than an average decrease of the mean distance between grains. The reorganization of the grains can be advantageously analyzed through the distribution of the pores volumes. It has been found on a consistent way with numerical simulations that compaction is mainly due to a decrease of the number of the largest pores. The volumes of these pores are statistically distributed along a broad exponential tail which progressively reduces while the structure gets more compact.

We are grateful to G. Vigier who is at the origin of this work. We acknowledge the European Synchrotron Radiation Facility (ESRF) for the use of their facilities, hospitality and financial help. We are indebted to J. T. Jenkins and G. Le Caër for a careful reading of the manuscript. Two of us (P.R. and P.P.) thank C. Menissez and S. Petitdidier for hospitality.
[1] S.F. Edwards and R.B.S. Oakeshott, Physica A, 157, 1080 (1989); A. Mehta and S.F. Edwards, Physica A 157, 1091 (1989).

[2] M. Nicodemi, A. Coniglio and H.J. Herrmann, Phys. Rev. E, 55, 3962 (1997); J. Kurchan, J. Phys: Cond. Matt., 12, 6611 (2000); A.J. Liu and S.R. Nagel, Nature, 396, 21 (1998).

[3] C. Josserand, A. Tkachenko, D.M. Mueth and H.M. Jaeger, Phys. Rev. Letters, 85, 3632 (2000).

[4] E. Caglioti, A. Coniglio, H.J. Herrmann and V. Loreto and M. Nicodemi, Physica A, 265, 311 (1999).

[5] J.B. Knight, C.G. Fandrich, C.N. Lau, H.M. Jaeger and S.R. Nagel, Phys. Rev. E, 51, 3957 (1995); E.R Nowak, J.B. Knight, E. Ben-Naim, H.M. Jaeger and S.R. Nagel, Phys. Rev. E 57, 1971 (1998).

[6] P. Philippe and D. Bideau, Europhys. Letters, 60, 677 (2002).

[7] J.C. Phillips, Rep. Prog. Phys., 59, 1133 (1996).

[8] G.T. Seidler, G. Martinez, L.H. Seeley, K.H. Kim, E.A. Behne, S. Zaranek, B.D. Chapman, S.M. Heald and D.L. Brewe, Phys. Rev. E, 62(6), 8175 (2000).

[9] M.E. Coles, Experimental Methods in the Physical Sci- ences, Edited by P.-Z.Wong, (Academic Press, Sans Diego, 1999), Vol. 35, pp. 301-336.

[10] G.T. Herman, Image Reconstruction from Projections. Academic Press, New York, 1980.

[11] F. Natterer, The mathematics of computerized tomography. John Wiley sons, New York, 1986.

[12] A. Rosato and D. Yacoub, Powder Technology, 109, 255 (2000).

[13] P. Richard, A. Gervois, L. Oger and J.-P. Troadec, Europhysics Letters 48, 415 (1999) and references therein.

[14] T. Boutreux and P.G. de Gennes, Physica A, 244, 59 (1997).

[15] P. Richard, L. Oger, J. Lemaitre, L. Samson and N.N. Medvedev, Granular Matter,1(4), 203 (1999).

[16] P. Philippe and D. Bideau, Phys. Rev. E, 63, 051304 (2001).

[17] P. Richard, L. Oger, J.P. Troadec and A. Gervois, Eur. Phys. Jour. E, 6, 295 (2001).

[18] E.R. Nowak, J.B. Knight, M.L. Povinelli, H.M. Jaeder and S.R. Nagel, Powder Technology, 94, 79 (1997). 